\title{
DEFORMABLE ALIGNMENT USING RANDOM PROJECTIONS OF LANDMARK IMAGES
}

\author{
Hui Wu ${ }^{\star}$, Dustin M. Bowers ${ }^{\star}$, Toan T. Huynh ${ }^{\dagger}$, and Richard Souvenir ${ }^{\star}$ \\ ${ }^{\star}$ Department of Computer Science, University of North Carolina at Charlotte \\ ${ }^{\dagger}$ Department of General Surgery, Carolinas Medical Center
}

\begin{abstract}
This paper presents a method for rigid alignment of objects undergoing deformation. Automated algorithms can be affected by auxiliary motion, such as image motion caused by transducer movement in echocardiography. Unlike deformable registration methods, the goal of this work is alignment without introducing additional distortion. Our method, based on random projection theory, incorporates motion metadata for phase-aware alignment and outperforms rigid alignment approaches on synthetic data. We demonstrate the benefit of this as a pre-processing step to two common biomedical image analysis tasks: object segmentation and video denoising.
\end{abstract}

Index Terms - echocardiography, alignment

\section{INTRODUCTION}

Biomedical video analysis is often complicated by the movement of the object of interest within the image plane. Whether the motion is internal (e.g., patient breathing) or external (e.g., transducer motion in ultrasound), it is often an impediment to later analysis (e.g., recognition, segmentation, shape estimation). Often the motion is "small enough" that it can be approximated as 2D rigid or affine motion.

Another complication is introduced when this auxiliary motion is accompanied by an uncorrelated motion, especially deformable motion. This is common in echocardiograms obtained from a critical care setting (Figure 1). The heart undergoes cyclic deformable motion while uncorrelated approximately rigid motion is introduced by the motion of the transducer and/or the patient breathing. The presence of deformable motion suggests that methods for non-rigid and deformable registration (e.g., [1]) would suffice; however, this is not the case. The deformable motion is typically the motion pattern of interest, so image warps applied to this data may affect inferences made on the motion patterns. In this paper, we present a method for rigid alignment of deformable objects that takes advantage of phase-correlated metadata commonly collected with medical images. We demonstrate how this approach, when used as a pre-processing step, improves biomedical image analysis tasks, such as segmentation and echocardiograms denoising.

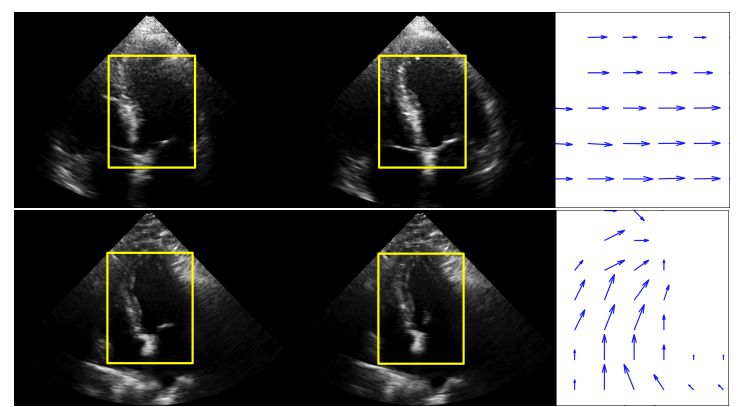

Fig. 1. Each row shows two echocardiogram frames and arrows indicating image motion. The top images vary due to sensor motion (rigid), while the bottom vary due to heart motion (deformable). Our algorithm aligns objects that undergo both types of motion simultaneously.

\section{RELATED WORK}

Registration is a widely-studied problem, and the literature is vast. Aligning an object of interest is a common pre-processing step for higher-level analysis, such as denoising [2], atlas construction [3], or segmentation [4].

Classic registration algorithms apply pairwise alignment through transforms of pairs of images in order to maximize some similarity measure. This general approach can be applied to both rigid (e.g., [5]) and non-rigid deformations (e.g., [1]). Recently, there has been increasing interest in group-wise image alignment [6]. Similar to our work, these methods assume that a single reference image is insufficient for alignment; most update the reference iteratively until convergence. Recent work makes use of the manifold relation among images in a set. In [7], all of the images are registered to the image with the closest geodesic distances to all other points. While this work applies the manifold assumption, it is used to register the images to a single reference.

In terms of methodology, the method most similar to our work is [8], where the manifold property of natural image sets is exploited to quickly align images of objects undergoing rigid transformation. We extend this method to incorporate the phase information of objects undergoing simultaneously uncorrelated deformable motion. 

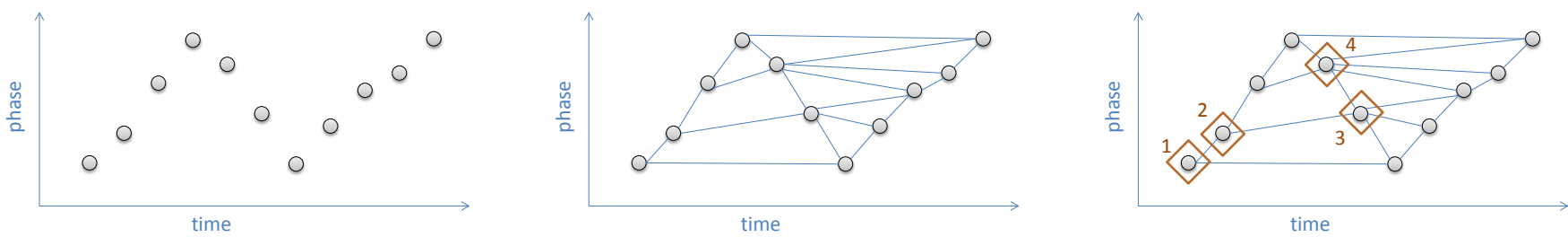

Fig. 2. Overview of landmark selection. (left) Each point represents an input frame plotted by time (x-axis) and phase (y-axis). (middle) Images that are close in time or phase are candidates for reference targets and are connected by an edge. (right) Landmark images are selected using a greedy approach.

\section{APPROACH}

Under the brightness constancy constraint, the image registration problem is commonly formulated as:

$$
\min _{\theta} \phi\left(I_{R}, I(f(\theta))\right)
$$

where $I_{R}$ is a reference image, $f$ is an image transformation with parameters $\theta$, and $\phi$ is a dissimilarity measure (e.g., $\mathrm{SSD})$. When extended to video, $\mathcal{I}=\left[\mathbf{I}_{1}, \ldots, \mathbf{I}_{N}\right]$, the reference image, $I_{R}$ is often selected from the set, and temporal smoothing is commonly added to prevent large changes in alignment parameters for consecutive frames.

For our problem, we are provided with real-valued metadata, $S=\left[\mathbf{s}_{1}, \ldots, \mathbf{s}_{N}\right]$, associated with the deformable motion. We use this to impose a neighborhood constraint between images with similar phase values (i.e., similar shape). Incorporating temporal smoothness and the neighborhood constraint leaves the following optimization:

$$
\min _{\Theta} \sum_{i} \sum_{j \in \mathcal{N}_{i}} \phi\left(I_{i}\left(\theta_{i}\right), I_{j}\left(f\left(\theta_{j}\right)\right)\right)+\beta \sum_{i}\left|\frac{\partial \theta_{i}}{\partial i}\right|
$$

where $\Theta=\left\{\theta_{1}, \ldots, \theta_{N}\right\}$ are the transformation parameters for the video frames, $\beta$ is a weight on the smoothing term, and $\mathcal{N}_{i}$ represents the set of images that are neighbors to image $i$. Even for simple transformation models and similarity metrics, this can be a nonlinear, non-convex optimization. In the remainder of this section, we describe our approximation algorithm for deformable object alignment, which relies on selecting a small subset of images, called landmarks to serve as reference images for alignment.

\subsection{Landmark Selection}

The goal of landmark selection is to find a set of images that could serve as references, so the whole video is aligned. Ideally, the set is small, yet spans the range of phase values. This can be framed as a graph problem where the neighborhood relation represents the pairwise suitability of images to serve as references for each other. That is, we construct a graph, with one vertex for each image, and an edge between vertices if they are neighbors. Starting from a reference image (vertex), on a connected graph, there exists a path, such that the vertices on that path are adjacent to all of the vertices in graph. That is, those vertices represent the set of landmark images that will serve as reference images for alignment. Figure 2 demonstrates the landmark selection process.

\subsection{Image Alignment Using Random Projections}

The set of landmark images could be used to align the remaining images. For efficient alignment, we employ the theory of random projection, which has been previously used to improve the speed of image alignment algorithms [8]. Briefly, random projection is based on the theory that when highdimensional data (e.g., images) are projected onto a random subspace (of sufficient dimensionality), the (dis)similarities between the data points are preserved. The benefit is that distances can be computed more quickly in lower-dimensional spaces and data structures (e.g., kd-trees) which are inefficient for high-dimensional data, can be used. In the next section, we combine landmark selection and alignment via random projections for our deformable object alignment algorithm.

\subsection{Algorithm}

Given an input video, $\mathcal{I}=\left[\mathbf{I}_{1}, \ldots, \mathbf{I}_{N}\right]$, metadata, $S=$ $\left[\mathbf{s}_{1}, \ldots, \mathbf{s}_{N}\right]$, and a neighborhood relation, $\mathcal{N}_{i}$ for each frame, our algorithm computes a set of corresponding rigid alignment parameters, $\Theta=\left\{\theta_{1}, \ldots, \theta_{N}\right\}$, as follows:

1. Construct a graph, $G$, with one vertex for each image, and an edge between vertices $v_{i}$ and $v_{j}$ if $j \in \mathcal{N}_{i}$.

2. Select a landmark image as the reference, $I_{R}$. (Either randomly or defined as input.)

3. Compute a random projection matrix, $P .{ }^{1}$

4. Project each image, $I_{i}$, and apply temporal smoothing to the embedded coordinates

5. Let $I_{L}$ be the current landmark image, initialized to $I_{R}$.

6. Until all the images are aligned:

(a) Transform $I_{L}$ across the range of transformation parameters and maintain the projected coordinates of each transformed instance.

\footnotetext{
${ }^{1}$ Due to space constraints, the details are omitted. However, a wide range of sparse random matrices will suffice.
} 

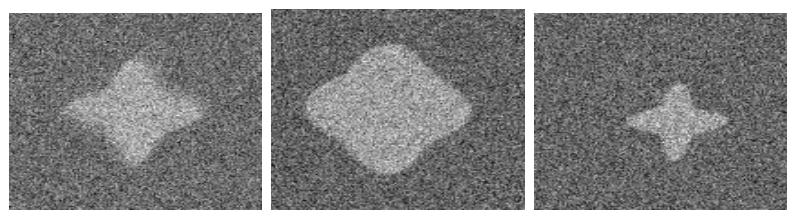

Fig. 3. Three synthetic video frames depicting the simultaneous deformable and rigid motions.

(b) For each image, $I_{j}$, adjacent to $I_{L}$, find the closest transformed instance, $I_{L}^{\prime}$. Set the alignment parameters of $I_{j}$ to those of $I_{L}^{\prime}$ plus $I_{L}$.

(c) Update $I_{L}$ to be the already aligned image adjacent to the most unaligned images.

Compared to full pairwise alignment, this greedy algorithm is efficient and examines each image only a single time. The most expensive step is 6(a), where the current landmark is transformed and projected across the space of transformations. This occurs once for each landmark image, and in Section 4, we demonstrate that the number of landmark images does not increase with the video length.

\section{RESULTS AND DISCUSSION}

Our evaluation consists of quantitative results on synthetic data and qualitative results on real-world data. For the synthetic data, we measure the alignment accuracy of our method compared to other approaches, and for the real-world data, we evaluated how much object segmentation and image denoising are improved after our method is applied as a preprocessing step. For all experiments, the neighborhood criteria corresponds to a $10 \%$ maximum difference in phase value.

\subsection{Synthetic Data}

The synthetic video set was constructed by applying a parametric non-rigid warp to an initial shape and translating the shape along an arbitrary path. Random Gaussian noise was then added to each frame. Figure 3 shows three sample frames from this data set. We compare the following approaches:

- BASE: baseline where each frame is aligned to a reference

- RP: single reference random projection method [8]

- LM: phase-aware landmark alignment (proposed)

For all the methods, the reference image was randomly selected. For the baseline approach, sum of squares pixel differences (SSD) was minimized. For all methods, error is computed as the SSD between the returned alignment parameters and the ground truth. Table 1 shows the average results for repeated experiments using all three methods. Error is reported as RMSE in pixel distances from the known ground truth location. For each trial, the motion patterns, noise level, and reference image were randomized. Overall, our method (LM) outperformed both methods.

\begin{tabular}{|l|c|}
\hline Method & RMSE \\
\hline BASE & $4.48 \pm .96$ \\
\hline RP & $3.71 \pm .84$ \\
\hline LM & $2.17 \pm .96$ \\
\hline
\end{tabular}

Table 1. RMSE results on synthetic video in pixel units.

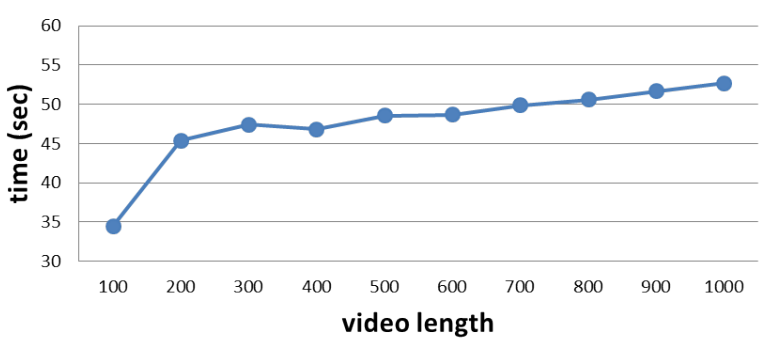

Fig. 4. Running time as a function of video length.

We measured the runtime, by varying the length of the input video. Figure 4 shows the amount of time (in seconds) as the number of frames increases. Similar to [8], the runtime is dominated by transforming the landmark images. However, the number of landmarks is determined by the data distribution and neighborhood criteria, not the number of frames.

\subsection{Applications to Echocardiography}

Even echocardiograms collected by trained technicians exhibit image motion due to transducer motion. These slight motions can be approximated by rigid image transformations. Here, we demonstrate how our method can be used to improve two common applications in echocardiography: segmentation and denoising. The data for these experiments consists of parasternal short axis (PSAX) echocardiograms and phase parameters extracted from the synchronized ECG signal using an automated signal processing algorithm [9].

\subsubsection{Left Ventricle Segmentation}

Left ventricle segmentation is the most common application in cardiac image analysis. We applied a method [4] that uses an adaptive diffusion flow active-contour model to (1) the original data and (2) after it was aligned by our method and reordered by the phase parameter. Except for the first frame, the contour for each frame was initialized using the final contour from the previous frame. Figure 5 shows the segmentation results on three sample frames using the original (top) and aligned (bottom) data. In the unaligned case, there are noticable instances of boundary leakage; most occur shortly after larger than normal object motion in the image. Additionally, the aligned case required fewer iterations of the method to reach convergence. 

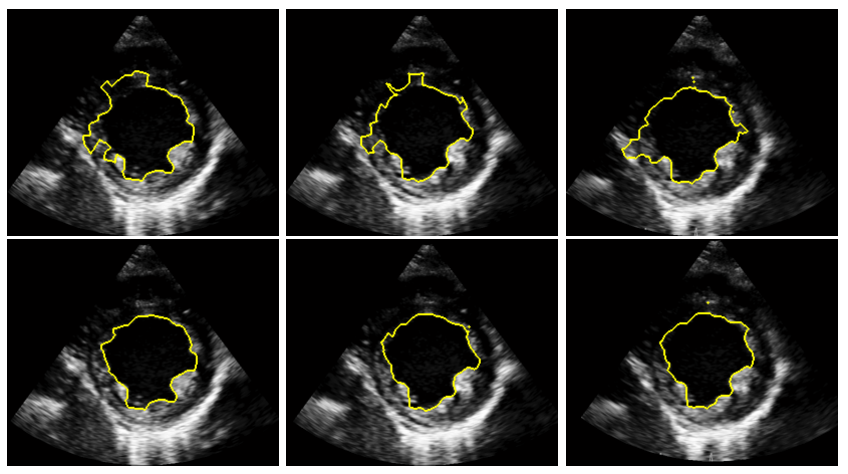

Fig. 5. Each column shows the boundary detected using an active contour method [4] on the original data (top) and after alignment with our method (bottom).
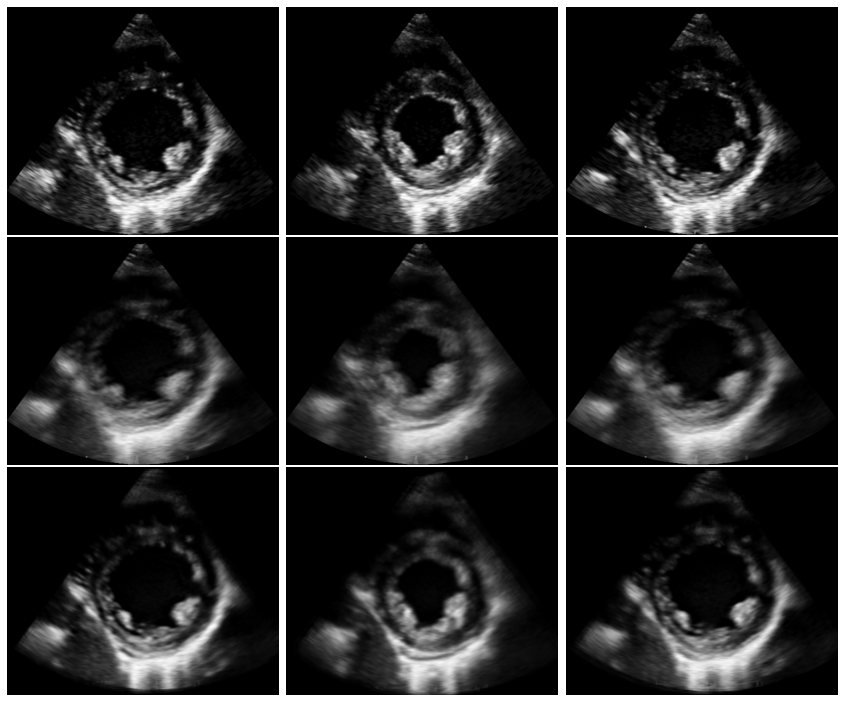

Fig. 6. Each column shows (top) an echocardiogram frame after phase-aware denoising on the (middle) original data and (bottom) after alignment with our method .

\subsubsection{Video Denoising}

A recent phase-aware video denoising method [2] uses supervised manifold learning to denoise biomedical video given associated phase-correlated metadata. By projecting the images to a manifold correlated with the phase metadata, this approach discards uncorrelated motion information. We applied this method to PSAX echocardiograms, and the results on three frames are shown in Figure 6. A visual comparison of the denoised output of the original data (middle) and aligned (bottom) shows significant differences. In the unaligned case, motion blur is visible in the denoised output and detail is lost.

\section{CONCLUSIONS AND FUTURE WORK}

We presented a method for phase-aware alignment of deformable objects. Cases of simultaneous rigid and non-rigid are prevalent, especially in echocardiography. The ultrasound data used in Section 4.2 were collected by an expert technician, yet it contained enough auxiliary motion to have an impact on automated processing algorithms. For future work, we plan to investigate better landmark selection schemes and methods for selective projection of transformed landmarks.

\section{REFERENCES}

[1] M. Holden, "A review of geometric transformations for nonrigid body registration," Medical Imaging, IEEE Transactions on, vol. 27, no. 1, pp. 111-128, 2008.

[2] H. Wu, D.M. Bowers, T.T. Huynh, and R. Souvenir, "Biomedical video denoising using supervised manifold learning," in International Symposium on Biomedical Imaging, 2013, pp. 1244-1247.

[3] A.F. Frangi, D. Rueckert, J.A. Schnabel, and W.J. Niessen, "Automatic construction of multiple-object three-dimensional statistical shape models: application to cardiac modeling," Medical Imaging, IEEE Transactions on, vol. 21, no. 9, pp. 1151-1166, 2002.

[4] Y. Wu, Y. Jia, and Y. Wang, "Adaptive diffusion flow for parametric active contours," in Proc. International Conference on Pattern Recognition, 2010, pp. 2788-2791.

[5] A. Roche, X. Pennec, G. Malandain, and N. Ayache, "Rigid registration of 3-d ultrasound with mr images: a new approach combining intensity and gradient information," Medical Imaging, IEEE Transactions on, vol. 20, no. 10, pp. 1038-1049, 2001.

[6] T.F. Cootes, C.J. Twining, V.S. Petrovic, K.O. Babalola, and C.J. Taylor, "Computing accurate correspondences across groups of images," Pattern Analysis and Machine Intelligence, IEEE Transactions on, vol. 32, no. 11, pp. 1994-2005, 2010.

[7] S. Ying, G. Wu, Q. Wang, and D. Shen, "Groupwise registration via graph shrinkage on the image manifold," in Proc. IEEE Conference on Computer Vision and Pattern Recognition, 2013, pp. 2323-2330.

[8] D.M. Healy and G.K. Rohde, "Fast global image registration using random projections," in International Symposium on Biomedical Imaging. IEEE, 2007, pp. 476-479.

[9] Q. Zhang, A.I. Manriquez, C. Medigue, Y. Papelier, and M. Sorine, "An algorithm for robust and efficient location of t-wave ends in electrocardiograms," Biomedical Engineering, IEEE Trans on, vol. 53, no. 12, pp. 2544 -2552, Dec 2006. 